\title{
Urogenital Microbiota:Potentially Important Determinant of PD-L1 Expression in Male Patients with Non-muscle Invasive Bladder Cancer
}

\author{
Chunxiao Chen ${ }^{1 \dagger}$, Zehai Huang ${ }^{1 \dagger}$, Pengcheng Huang ${ }^{1}$, Kun Li ${ }^{2}$, Jiarong Zeng ${ }^{3}$, Yuehui Wen ${ }^{4}$, Biao $\mathrm{Li}^{1}$,
} Jie Zhao ${ }^{5^{*}}$ and Peng $\mathrm{Wu}^{1,6^{*}}$

\begin{abstract}
Background: Urogenital microbiota may be associated with the recurrence of bladder cancer, but the underlying mechanism remains unclear. The notion that microbiota can upregulate PD-L1 expression in certain epithelial tumors to promote immune escape has been demonstrated. Thus, we hypothesized that the urogenital microbiota may be involved in the recurrence and progression of non-muscle invasive bladder cancer (NMIBC) by upregulating the PD-L1 expression. To test this hypothesis, we investigated the relationship between urogenital microbial community and PD-L1 expression in male patients with NMIBC.

Results: $16 \mathrm{~S}$ rRNA gene sequencing was performed to analyse the composition of urogenital microbiota, and the expression of PD-L1 in cancerous tissues was detected by immunohistochemistry. The subjects (aged 43-79 years) were divided into PD-L1-positive group (Group P, $n=9$ ) and PD-L1-negative group (Group N, $n=19$ ) respectively based on their PD-L1 immunohistochemical results. No statistically significant differences were found in the demographic characteristics between group P and N. We observed that group P exhibited higher species richness (based on Observed species and Ace index, both $P<0.05$ ). Furthermore, subgroup analysis showed that the increase in number of PD-L1 positive cells was accompanied by increased richness of urogenital microbiota. Significantly different composition of urogenital microbiota was found between group P and group N (based on weighted Unifrac and unweighted Unifrac distances metric, both $P<0.05$ ). Enrichment of some bacterial genera (e.g., Leptotrichia, Roseomonas, and Propionibacterium) and decrease of some bacterial genera (e.g., Prevotella and Massilia) were observed in group P as compared with group N. These findings indicated that these genera may affect the expression of PD-L1 through some mechanisms to be studied.
\end{abstract}

Conclusion: Our study provided for the first time an overview of the association between urogenital microbiota and PD-L1 expression in male patients with NMIBC, indicating that urogenital microbiota was an important determinant of PD-L1 expression in male NMIBC patients.

\footnotetext{
*Correspondence: zhaojie_0412@163.com; doctorwupeng@gmail.com

${ }^{\dagger}$ Chunxiao Chen and Zehai Huang contributed equally to this work.

${ }^{5}$ School of Pharmaceutical Sciences, Southern Medical University, Guangzhou, China

${ }^{6}$ Clinical Microbiota Center, Nanfang Hospital, Southern Medical

University, Guangzhou, China

Full list of author information is available at the end of the article
}

(C) The Author(s) 2021. Open Access This article is licensed under a Creative Commons Attribution 4.0 International License, which permits use, sharing, adaptation, distribution and reproduction in any medium or format, as long as you give appropriate credit to the original author(s) and the source, provide a link to the Creative Commons licence, and indicate if changes were made. The images or other third party material in this article are included in the article's Creative Commons licence, unless indicated otherwise in a credit line to the material. If material is not included in the article's Creative Commons licence and your intended use is not permitted by statutory regulation or exceeds the permitted use, you will need to obtain permission directly from the copyright holder. To view a copy of this licence, visit http://creativecommons.org/licenses/by/4.0/. The Creative Commons Public Domain Dedication waiver (http://creativeco mmons.org/publicdomain/zero/1.0/) applies to the data made available in this article, unless otherwise stated in a credit line to the data. 
Keywords: Bladder cancer, Urogenital tract, Microbiota, PD-L1, Immune escape

\section{Background}

Bladder cancer, with an estimated 549,000 new cases and 200,000 deaths according to a 2018 report on the incidence and mortality of 36 cancers in 185 countries, ranked 10th among the most common malignancies worldwide [1]. And about $75 \%$ of bladder cancers are non-muscle invasive bladder cancer (NMIBC) at initial presentations, among which, Ta and T1 lesions are generally characterized with a good prognosis and low lethal potential [2], but, their 5-years recurrent rates are $50-70 \%$ after initial treatments, and $10-30 \%$ of which will even progress into muscle-invading tumor [3]. Researchers have long been studying the recurrence causes of non-muscle invasive bladder cancer, unfortunately, the exact causes and mechanisms remain unclear.

Some studies have shown that immune escape might be involved in the recurrence and progression of bladder cancer via the PD-L1/PD1 pathway $[4,5]$. PD-L1 (B7H1) is a member of the B7 family of costimulatory molecules. It mainly promotes apoptosis by combining with programmed death receptors (PD1) expressed on the surface of $T$ cells and $B$ cells, thereby suppressing host immune function and achieving tumor immune escape $[6,7]$. Hurwitz ME et al. reported that PD-L1 expression appears to increase as bladder cancer recurs [5]. The recurrence and progression rates of NMIBC are higher in patients with positive PD-L1 expression than that in patients with negative PD-L1 expression. And various factors and mechanisms are involved in the regulation of PD-L1 expression, such as IFN $\gamma$ and microbiota [8-13]. Some researches have demonstrated that microbiota can upregulate the expression of PD-L1 in certain epithelial tumors and allergic diseases to promote immune escape or immune tolerance [11-13]. But, whether the microbiota in urogenital tract have the similar impact on PD-L1 expression in NMIBC remains unknown.

The human microbiota is the full collection of microbes (bacteria, fungi, parasites, viruses, etc.) that inhabit the epithelial barrier surfaces of our body [14], the microbiota data in this study were obtained by analyzing the mid-stream voided urines, thus the term "urogenital microbiota" was used [15]. In recent years, the relationship between urogenital microbiota and bladder cancer has received widespread attention. It is reported that tobacco smoking, carcinogen exposure and Schistosomiasis are the common predisposing factors of bladder cancer [16]. Besides, an association between chronic bladder infection with Schistosoma haematobium and the subsequent development of bladder squamous cell carcinoma in both men and women has been long-recognized [17], while Hicks RM et al. reported that strains of bacteria may contribute to schistosomiasis-induced bladder cancer by mediating the formation of N-nitrosamines [18, 19]. Bacillus Calmette-Guerin (BCG), the Mycobacterium bovis-derived vaccine strain for tuberculosis, is widely used to prevent recurrence of bladder cancer by direct bladder instillation [20]. Since commensal microorganisms are also present in the bladder, they may potentially interact with BCG, influencing the efficacy of BCG [21]. Our previous study found that Herbaspirillum, Porphyrobacter and Bacteroides are enriched in bladder cancer patients with a high risk of recurrence and progression, which suggests that these genera might be associated with the recurrence of bladder cancer [22]. Zitvogel et al. reported that oral administration of Lactobacillus after removal of the bladder tumor could reduce the probability of recurrence [23], which added evidence to the relationship between microbiota and bladder cancer recurrence. The composition of urogenital microbiota is closely related to tobacco smoking, which indicates tobacco smoking may also promote the occurrence and development of bladder cancer by changing urogenital microbiota [24]. However, the underlying mechanism of urogenital microbiota affecting the recurrence of NMIBC remains to be further studied. The important roles of microbiota in multiple systems of the human body have been proven, including the immune system [25]. Therefore, based on the potential relationship between microbiota and PD-L1 expression, we hypothesized that the urogenital microbiota may be involved in the recurrence and progression of NMIBC by up-regulating PD-L1 expression.

In this study, we mainly aimed to assess the association between urogenital microbiota and PD-L1 expression in male patients with NMIBC and to screen for some potential taxa related to PD-L1 expression. The results can serve as a resource for further researches to gain a better understanding of the role of urogenital microbiota in tumor recurrence and identify candidate biomarkers for the application of PD1 or PD-L1 blockers in bladder cancer.

\section{Results}

\section{PD-L1 Expression on Tumor Cell Membrane or Immune} Cells

$16 \mathrm{~S}$ rRNA gene sequence data of 28 male patients with NMIBC were obtained, and their tumor samples were used to detect PD-L1 expression on tumor cells 

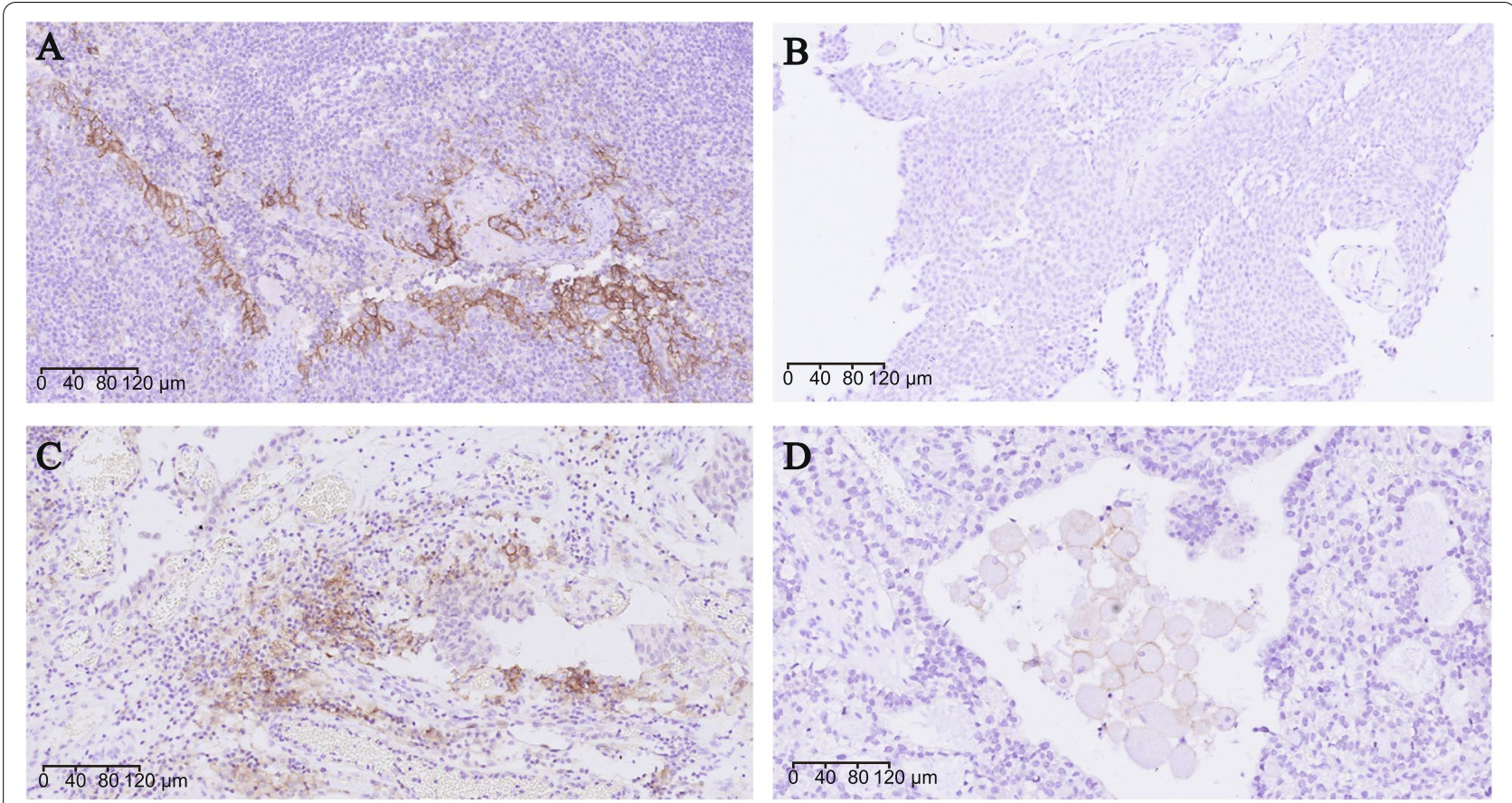

Fig. 1 PD-L1 expression in formalin-fixed paraffin-embedded tissue samples stained with anti-PD-L1 antibody (405.9A11). Positive control with tonsil tissue for PD-L1 expression is presented in panel (A). In panel (B), both tumor cells and immune cells are negative for PD-L1. Positive membranous staining in tumor cells and immune cells are presented in panels (C) and (D), respectively

or immune cells. Among them, the tumor stage of 13 patients was TaN0M0, while 15 patients was T1N0M0. Overall, PD-L1 expression was negative in 19 patients $(67.9 \%)$ and positive in 9 patients $(32.1 \%$, including 6 patients with PD-L1 expression on tumor cells and 3 patients on immune cells). And PD-L1 did not appear in tumor cells and immune cells simultaneously in PD-L1 positive specimens. Out of the 28 male patients with NMIBC, the intensity of PD-L1 was assessed and recorded as absent $(0)$ in 19 patients $(67.9 \%)$, mild $(1+)$ in 6 patients $(21.4 \%)$, moderate $(2+)$ in 1 patient $(3.6 \%)$, and severe (3+) in 2 patients (7.1\%). Representative immunohistochemical staining results are shown in Fig. 1.

\section{Demographic Characteristics of Subjects}

A total of 31 mid-stream urine specimens were analyzed, including 11 from male NMIBC patients with positive PD-L1 expression and 20 from male NMIBC patients with negative PD-L1 expression, while 2 specimens in PD-L1-positive group and 1 specimen in PD-L1-negative group were excluded for too little sequencing reads. Finally, 28 male patients with NMIBC based on their PD-L1 immunohistochemical results were divided into PD-L1-positive group (group P) and PD-L1-negative group (group N), respectively. No statistically significant differences were found in the demographic characteristics between group P and N (Table 1).

\section{Sequencing Data, Alpha, Beta Diversity and Alpha Rarefaction Curves}

Reads that contain over $50 \%$ of base with quality value less than 20 or that contain over $65 \%$ of base with quality value less than 30 were removed. A total of 962,452 reads were obtained from 28 samples after the removal of primer and chimera. The median number of reads in PD-L1 positive group was 26,335 , and in the PD-L1 negative group was $34,952(P=0.676)$. And the reads were classified into 2,124 OTUs which were used for downstream analysis. More OTUs were identified in urine samples from group P, with an average of 215 OTUs per sample in group $\mathrm{P}$ and 141 OTUs per sample in group $\mathrm{N}(P=0.029)$. The number of reads and OTUs for each sample are shown in Supplementary Table S1. The Ace index, Observed species and Rarefaction curves all shown that the urogenital microbiota in group $\mathrm{P}$ was much richer than that in group N. $(P<0.05$; Figs. $2 \mathrm{~A}$, $\mathrm{B}$ and $2 \mathrm{~F})$. While no difference was found in the Chao1 index (Fig. 2C), Shannon index (Fig. 2D) and Simpson index (Fig. 2E) between group $\mathrm{P}$ and group $\mathrm{N}$.

In order to assess the relationship between PD-L1 expression intensity and urogenital microbiota, we divided group P into group $C$ (with mild PD-L1 expression) and group $\mathrm{D}$ (with moderate or severe PD-L1 expression) based on the expression intensity of PD-L1. However, no statistically significant difference in the 
Table 1 Comparisons of demographic characteristics between PD-L1-positive (group P) and PD-L1-negative groups (group N)

\begin{tabular}{|c|c|c|c|}
\hline $\begin{array}{l}\text { Demographic } \\
\text { characteristics }\end{array}$ & Group P (n=9) & Group N ( $n=19)$ & $P$-value \\
\hline Age (y) & $67.11(11.48)^{\mathrm{a}}$ & $62.05(9.79)^{\mathrm{a}}$ & 0.238 \\
\hline Weight (kg) & $63.92(9.03)^{\mathrm{a}}$ & $64.79(7.96)^{\mathrm{a}}$ & 0.798 \\
\hline Height (m) & $1.66(0.06)^{a}$ & $1.68(0.05)^{\mathrm{a}}$ & 0.270 \\
\hline $\mathrm{BMI}\left(\mathrm{kg} / \mathrm{m}^{2}\right)$ & $23.16(2.00)^{\mathrm{a}}$ & $22.89(2.76)^{\mathrm{a}}$ & 0.794 \\
\hline Drinking history & $11.11 \%(1 / 9)$ & $21.05 \%(4 / 19)$ & 1.000 \\
\hline Hypertension & $44.44 \%(4 / 9)$ & $31.58 \%(6 / 19)$ & 0.677 \\
\hline Diabetes & $33.33 \%(3 / 9)$ & $10.53 \%(2 / 19)$ & 0.290 \\
\hline Hyperlipemia & $22.22 \%(2 / 9)$ & $0.00 \%(0 / 19)$ & 0.095 \\
\hline $\mathrm{CHD}$ & $22.22 \%(2 / 9)$ & $10.53 \%(2 / 19)$ & 0.574 \\
\hline $\mathrm{FHC}$ & $0.00 \%(0 / 9)$ & $5.26 \%(1 / 19)$ & 1.000 \\
\hline Smoking index & $200(0,1500)$ & $400(0,800)$ & 0.841 \\
\hline Percentage of smokers & $55.56 \%(5 / 9)$ & $73.68 \%(14 / 19)$ & 0.407 \\
\hline $\begin{array}{l}\text { Percentage of multiple } \\
\text { tumors }\end{array}$ & $22.22 \%(2 / 9)$ & $52.63 \%(10 / 19)$ & 0.223 \\
\hline $\begin{array}{l}\text { Percentage of } \\
\text { hypergrading }\end{array}$ & $77.78 \%(7 / 9)$ & $42.11 \%(8 / 19)$ & 0.114 \\
\hline EORTC_R & $4.44(2.79)^{a}$ & $4.37(2.83)^{\mathrm{a}}$ & 0.947 \\
\hline Percentage of HER & $55.56 \%(5 / 9)$ & $42.11 \%(8 / 19)$ & 0.689 \\
\hline EORTC_P & $8.89(4.78)^{\mathrm{a}}$ & $6.63(5.36)^{\mathrm{a}}$ & 0.292 \\
\hline Percentage of HEP & $66.67 \%(6 / 9)$ & $47.37 \%(9 / 19)$ & 0.435 \\
\hline $\begin{array}{l}\text { Percentage of high } \\
\text { invasive risk }\end{array}$ & $88.89 \%(8 / 9)$ & $63.16 \%(12 / 19)$ & 0.230 \\
\hline $\begin{array}{l}\text { Percentage of } \\
\text { T1N0M0 }\end{array}$ & $77.78 \%(7 / 9)$ & $42.11 \%(8 / 19)$ & 0.114 \\
\hline
\end{tabular}

Data are presented as mean (SD) or median (first quartile to the third quartile) for continuous variables or $\mathrm{n}(\%)$ for counting data

${ }^{a}$ Indicates that the datum is subject to normal distribution and homogeneity of variance test and is presented as mean (SD). Multiple tumors refer to the number of bladder tumor $\geq 2$. Abbreviations: BMI, body mass index; CHD, coronary atherosclerotic heart disease; FHC, family history of cancer; EORTC, European Organization Research and Treatment of Cancer Scoring system, R refer to recurrence and_P refer to progression; HER, recurrence score of EORTC $\geq 5$; HEP, progression score of EORTC $\geq 7$

bacterial richness was found between the two groups (Supplementary Figure S1). Among PD-L1 positive group, urogenital microbiota of patients with $\geq 5 \%$ tumor cells membrane staining had higher richness than that of patients with $<5 \%(P<0.05$; Supplementary Figure S2).

To measure the similarity of urogenital microbial communities between group $\mathrm{P}$ and group $\mathrm{N}$, we performed the unconstrained principal coordinate analysis (PCoA) based on weighted UniFrac and unweighted UniFrac distance metrics. The PCoA plots revealed that the composition of urogenital microbiota in group $\mathrm{P}$ differed from that in group $\mathrm{N}(P<0.05$ and $P=0.047$ for weighted UniFrac and unweighted UniFrac distances, respectively. Figs. 3A, B). Besides, we detected that 643 OTUs were enriched in group P, while 934 OTUs in group N, and 547 OTUs were shared between the two groups (Fig. 3C).

\section{Relative Abundance of Urogenital Bacteria in group $\mathrm{P}$ and group $\mathbf{N}$}

At phylum level, the urogenital microbiota was dominated by Firmicutes (22.8\% in group P, $34.4 \%$ in group N) and Proteobacteria (21.1\% in group P, $25.8 \%$ in group N), followed by Actinobacteria (6.6\% in group P, $4.0 \%$ in group $\mathrm{N}$ ) and Bacteroidetes (1.1\% in group P, 9.7\% in group $\mathrm{N}$ ) (Table 2, Fig. 4A). The urogenital microbial compositions of different groups at class, order, family and genus levels were exhibited in Figs. 4B-E. The genera compositions of all samples were exhibited in Fig. 4F. Though the relative abundance of so many microbes at various taxonomic levels differed between group $\mathrm{P}$ and group $\mathrm{N}$, only five taxa including Bacteroidetes $(P=0.017)$, Bacteroidia $(P$ $=0.022)$, Bacteroidales $(P=0.025)$, Prevotellaceae $(P=$ $0.028)$ and Prevotella $(P=0.04)$ were present with higher relative abundance in group $\mathrm{N}$ and one genus called Leptotrichia had higher abundance in group $\mathrm{P}(P=0.001)$, when the Metastats algorithm was used for comparison of the relative abundance of bacteria and the relative abundance threshold of microbes was set at $0.1 \%$ (Table 2).

\section{Specific Taxa Associated With PD-L1 Expression}

The specific microbial taxa associated with PD-L1 expression were identified by using LEfSe algorithm. The results showed that Corynebacteriale, Corynebacteriaceae, Corynebacterium_1, uncultured_Corynebacterium_sp, Propionibacterium, Dermabacteraceae, Brachybacterium, Roseomonas, Rhodospirillaceae, Pigmentiphaga were present at significantly higher compositional abundances in group $\mathrm{P}$ compared with group $\mathrm{N}$, whereas the relative abundance of Massilia, Oxalobacteraceae, Firmicutes were higher in group N (Figs. 5A, B). Heat tree was used to illustrate the taxonomic differences between group $\mathrm{N}$ and group P (Fig. 5C). Similarly, in group P, Roseomonas was enriched in the group with higher PD-L1 expression (group F, PD-L1 $\geq 5 \%$ ) and Prevotella had higher abundance in the group with lower PD-L1 expression (group O, $1 \% \leq$ PD-L1 < 5\%) (Supplementary Figure S3).

\section{Discussion}

Over the past decade, the microbiota in different parts of the human body have been characterized and some microbiota-related mechanisms which can directly impact the human health have begun to be identified [26]. Developing diagnostics and therapeutics that utilize those connections between the microbiome and the human health is the next frontier for translational microbiome science [27]. However, the falsifiable hypothesis needs to be discovered through observational studies 


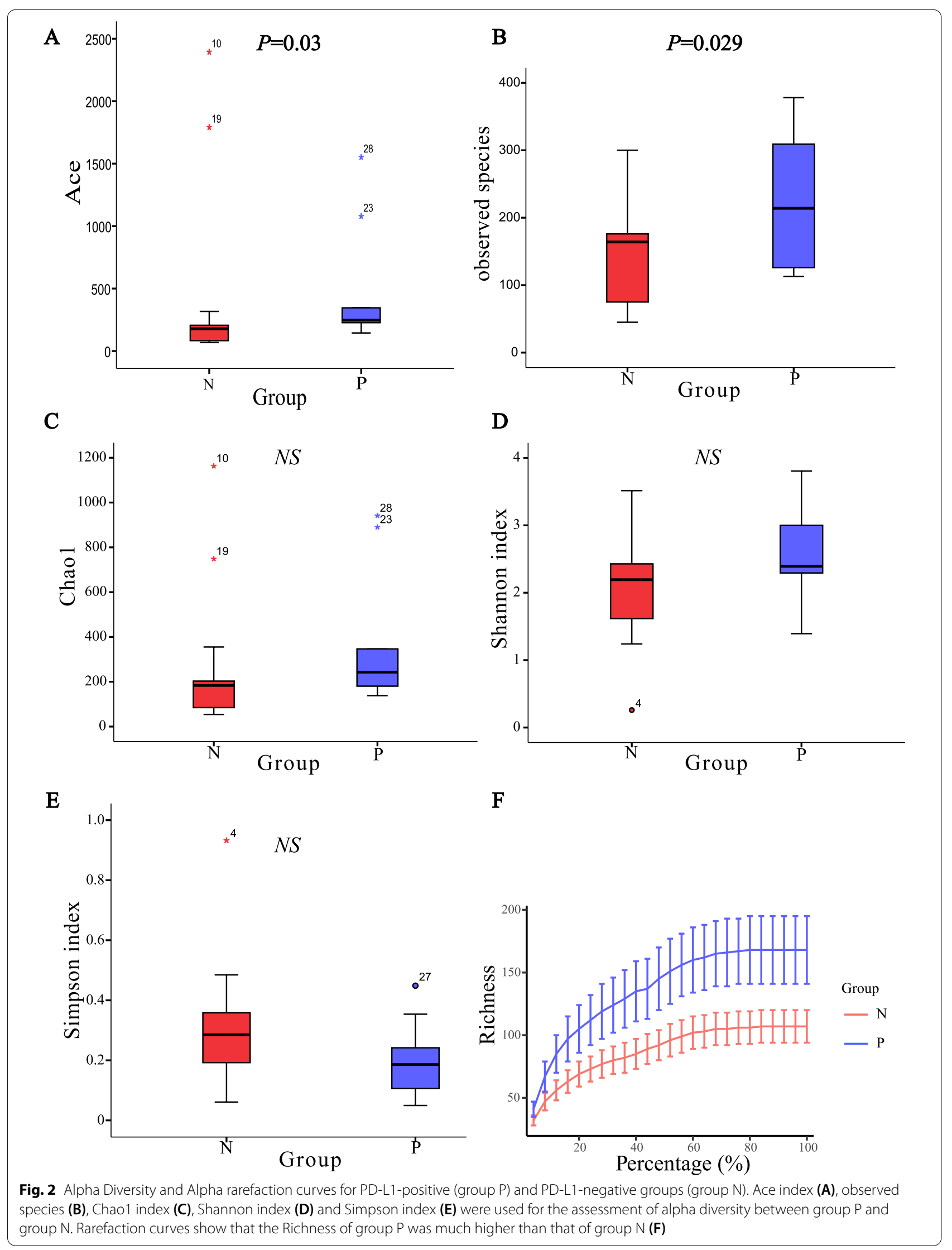



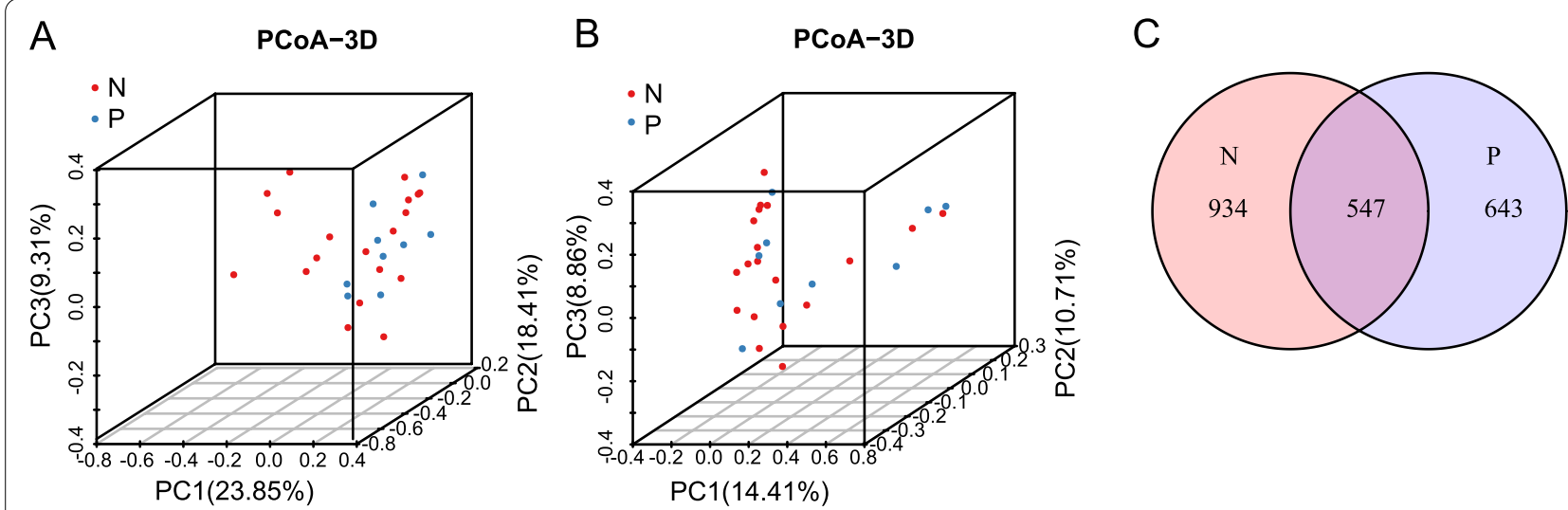

Fig. 3 The composition of urogenital microbiota in group P differed from that in group N. Principal coordinate analysis (PCoA) of weighted UniFrac distance (A) and unweighted UniFrac distance (B) show that the composition of urogenital microbiota in group P differed from that in group N $(P$ $<0.05$ and $P=0.047$ for weighted UniFrac and unweighted UniFrac distances, respectively). Venn diagrams (C) depict different OTU compositions between group $\mathrm{N}$ and group $\mathrm{P}$

before performing a randomized validation experiment. In this exploratory study, we found the associations between urogenital microbiota and PD-L1 expression in patients with NMIBC and some genera related to PD-L1 expression which may be isolated by bacterial culture technique and experimentally verified for their roles in regulating the expression of PD-L1. The findings of this study can serve as a resource for further researches to gain a better understanding of the roles of urogenital microbiota in tumor recurrence and identify candidate biomarkers for the application of PD1 or PD-L1 blockers in bladder cancer.

The role of PD-L1/PD1 mediated immune escape in bladder cancer recurrence and progression has long been studied $[4,5]$, which has facilitated the development of clinical trials of PD-L1/PD1 inhibitors in NMIBC and the FDA's approval of 5 PD-L1/PD1 inhibitors for the treatment of metastatic bladder cancer [28]. And in order to improve the response rate and reduce drug-related adverse reactions, patients with bladder cancer often receive PD-L1/PD1 inhibitors therapy depending on their PD-L1 status [29], which indicates the PD-L1 expression level is still one of the main factors that determine the efficacy of PD1/PD-L1 inhibitors. Therefore, exploring factors affecting the expression of PD-L1 may help improve the therapeutic effect of PD-L1/PD1 inhibitors. Groeger S et al. have reported that Porphyromonas gingivalis and its total membrane fraction can upregulate PD-L1 expression in vitro [11, 12]. In addition, microbiota could promote immune tolerance to allergens in neonates via PD-L1 [13]. And the concept that microbiota can modulate the clinical response to PD1/PD-L1 inhibitors has been confirmed in experimental studies [30,31], though the underlying mechanisms are still unknown.
Previous studies and ours have demonstrated the correlation between the microbiota and PD-L1 expression, suggesting that the microbiota may affect the therapeutic effect of PD1/PD-L1 inhibitors by regulating PD-L1 expression. If the roles of urogenital microbiota in PD-L1 expression would be confirmed in the future, urogenital microbiota might be a target for enhancing clinical responses to PD1/PD-L1 inhibitors in the treatment of bladder cancer or other urogenital tract tumors.

Increased microbial richness was observed both in muscle-invasive bladder cancer patients and NMIBC patients with a high risk of recurrence and progression [22], which was consistent with the results of Hai Bi group [32]. In present study, significantly higher bacterial richness in the PD-L1 positive group was found as well, which suggests that higher bacterial richness may be combined with PD-L1 or used as a substitute for PD-L1 to predict the recurrence or progression of NMIBC. Despite the inter-individual differences in the microbiota composition, the urogenital microbiota of individuals in the same group still have similarities, as shown in the PCoA analysis that clustered PD-L1 positive group and PD-L1 negative group separately (Figs. $3 \mathrm{~A}$ and $3 \mathrm{~B}$ ), indicating the possibility of a common urogenital microbiota disorder associated with PD-L1 expression.

In our study, we found that the Leptotrichia was enriched in PD-L1 positive male patients with NMIBC and Prevotella was enriched in PD-L1 negative group. Fusobacterium and Leptotrichia are closely-related organisms. They have been isolated from periodontal lesions and a diversity of genitourinary and gastrointestinal abscesses, and have been suggested to be potential emerging pathogens [33]. Studies have demonstrated that Leptotrichia is involved in the occurrence and 
Table 2 Comparison of relative abundance of urogenital microbiota between PD-L1 positive (group P) and PD-L1 negative groups (group N)

\begin{tabular}{|c|c|c|c|c|}
\hline Taxa & & Group N & Group P & $P$-value \\
\hline Phylum & $\begin{array}{l}\text { Firmicutes } \\
\text { Proteobacteria } \\
\text { Bacteroidetes } \\
\text { Actinobacteria }\end{array}$ & $\begin{array}{l}34.438 \\
25.756 \\
9.662 \\
4.012\end{array}$ & $\begin{array}{l}22.828 \\
21.077 \\
1.130 \\
6.631\end{array}$ & $\begin{array}{l}\text { Ns } \\
\text { Ns } \\
0.017 \\
\text { Ns }\end{array}$ \\
\hline Class & $\begin{array}{l}\text { Bacteroidia } \\
\text { Betaproteobacteria } \\
\text { Negativicutes } \\
\text { Actinobacteria } \\
\text { Bacilli } \\
\text { Unclassified } \\
\text { Gammaproteobacteria } \\
\text { Alphaproteobacteria }\end{array}$ & $\begin{array}{l}9.334 \\
4.557 \\
3.230 \\
3.085 \\
27.572 \\
22.313 \\
19.051 \\
1.765\end{array}$ & $\begin{array}{l}0.856 \\
2.234 \\
2.353 \\
6.488 \\
19.562 \\
44.950 \\
16.725 \\
2.012\end{array}$ & $\begin{array}{l}0.022 \\
\text { Ns } \\
\text { Ns } \\
\text { Ns } \\
\text { Ns } \\
\text { Ns } \\
\text { Ns } \\
\text { Ns }\end{array}$ \\
\hline Order & $\begin{array}{l}\text { Enterobacteriales } \\
\text { Bacteroidales } \\
\text { Pseudomonadales } \\
\text { Pasteurellales } \\
\text { Burkholderiales } \\
\text { Selenomonadales } \\
\text { Unclassified } \\
\text { Lactobacillales } \\
\text { Bacillales } \\
\text { Corynebacteriales }\end{array}$ & $\begin{array}{l}9.744 \\
9.316 \\
5.051 \\
3.973 \\
3.477 \\
3.226 \\
23.076 \\
16.826 \\
10.619 \\
1.928\end{array}$ & $\begin{array}{l}6.043 \\
0.853 \\
6.358 \\
1.995 \\
2.042 \\
2.352 \\
46.121 \\
11.731 \\
7.522 \\
4.426\end{array}$ & $\begin{array}{l}\text { Ns } \\
0.025 \\
\text { Ns } \\
\text { Ns } \\
\text { Ns } \\
\text { Ns } \\
\text { Ns } \\
\text { Ns } \\
\text { Ns } \\
\text { Ns }\end{array}$ \\
\hline Family & $\begin{array}{l}\text { Enterobacteriaceae } \\
\text { Prevotellaceae } \\
\text { Staphylococcaceae } \\
\text { Unclassified } \\
\text { Pasteurellaceae } \\
\text { Veillonellaceae } \\
\text { Moraxellaceae } \\
\text { Comamonadaceae } \\
\text { Pseudomonadaceae } \\
\text { Streptococcaceae } \\
\text { Corynebacteriaceae }\end{array}$ & $\begin{array}{l}8.580 \\
7.980 \\
7.876 \\
31.822 \backslash \\
3.966 \\
2.603 \\
2.510 \\
2.452 \\
2.335 \\
10.553 \\
1.788\end{array}$ & $\begin{array}{l}6.001 \\
0.761 \\
7.288 \\
46.981 \\
1.995 \\
2.347 \\
5.147 \\
1.804 \\
1.117 \\
10.664 \\
4.296\end{array}$ & $\begin{array}{l}\text { Ns } \\
0.028 \\
\text { Ns } \\
\text { Ns } \\
\text { Ns } \\
\text { Ns } \\
\text { Ns } \\
\text { Ns } \\
\text { Ns } \\
\text { Ns } \\
\text { Ns }\end{array}$ \\
\hline Genus & $\begin{array}{l}\text { Escherichia-Shigella } \\
\text { Prevotella } \\
\text { Staphylococcus } \\
\text { Unclassified } \\
\text { Haemophilus } \\
\text { Pseudomonas } \\
\text { Streptococcus } \\
\text { Delftia } \\
\text { Veillonella } \\
\text { Enhydrobacter } \\
\text { Leptotrichia }\end{array}$ & $\begin{array}{l}8.167 \\
8.060 \\
7.187 \\
36.701 \\
3.899 \\
2.306 \\
10.433 \\
1.817 \\
1.727 \\
1.332 \\
0.000\end{array}$ & $\begin{array}{l}4.840 \\
0.672 \\
6.941 \\
49.489 \\
1.983 \\
1.106 \\
10.485 \\
1.475 \\
2.213 \\
3.560 \\
0.205\end{array}$ & $\begin{array}{l}\text { Ns } \\
0.040 \\
\mathrm{Ns} \\
\mathrm{Ns} \\
\mathrm{Ns} \\
\mathrm{Ns} \\
\mathrm{Ns} \\
\mathrm{Ns} \\
\mathrm{Ns} \\
\mathrm{Ns} \\
0.001\end{array}$ \\
\hline
\end{tabular}

Data were displayed as mean percentage; For taxa with $P$-value $<0.05$, the taxa shown in the table are those with relative abundance greater than $0.1 \%$. For taxa without significant difference, only taxa with relative abundance greater than $1 \%$ are shown in the table; $N$ s, not significant (based on $P<0.05$ )

development of several carcinomas, such as gastric cancer, colon cancer, pancreatic cancer and etc [34-38]. Jang et al. reported IL- 6 and IL- 8 were strongly induced by Leptotrichia wadei [39], and IL6 is an inflammatory cytokine involved in various biological processes, including immune disorders and cancers [40, 41]. Chan et al. have demonstrated that Pro-inflammatory cytokines such as IFNy and IL-6, could promote the expression of
PD-L1 to achieve tumor immune escape [8, 42], which is consistent with our results. However, whether Leptotrichia can induce the expression of PD-L1 in bladder cancer and its related mechanisms need further confirmation in vivo and in vitro. As for Prevotella, Calcinotto A and collaborators recently reported that the abundance of Prevotella histicola and Prevotella melaninogenica in the guts of preclinical models and the humans or their colonization of the mouse guts are associated with decreased proinflammatory Th17 cells in the intestine and bone marrow, leading to delayed multiple myeloma progression [43]. On the contrary, Prevotella heparinolytica accelerated the progression of multiple myeloma in mice by inducing the local differentiation of Th17 cells that migrated to the bone marrow and supported plasma cells' survival and Prevotella spp. could sustain the protumorigenic effect of dextran sodium sulfate by favoring Th17 and Th1 immunity in the colon as well [43, 44]. Taken together, data from malignancy patients and murine experimental models converge to support the perspective that Prevotella may participate in the progression of malignant tumors through Th17 cells, but different strains of Prevotella may play opposite roles.

Roseomonas and Propionibacterium were found in higher abundances in PD-L1 positive group than in PD-L1 negative group. Roseomonas mucosa may directly influence atopic dermatitis and provide clinical benefit through multiple mechanisms, including innate/adaptive immune balance [45]. While Propionibacterium strain, P. UF1, can safeguard against proinflammatory diseases by increasing the frequencies of colonic Th17 and Treg cells [46]. These studies, together with ours, indicate that these two genera may affect the course of human diseases by regulating host's immunity.

This study does have limitations. First of all, the causal relationship between the urogenital microbiota and PD-L1 expression can not be determined by this observational study, which requires further prospective studies and experiments in vivo and in vitro to verify. Secondly, although it is a far less invasive method than suprapubic aspiration or catheterization and therefore is suitable for daily practice, midstream urine specimens collected by the clean catch method may be contaminated by microbiota in the urethra or surrounding the urethral orifice. Last but not least, 16S rRNA gene sequencing may reliably characterize microbiota to the genus level but cannot identify bacteria well at species level and detect viruses or fungi. However, its low cost and easy sampling make $16 \mathrm{~S}$ rRNA gene sequencing always a pilot in metagenome and other microbiota-related researches.

\section{Conclusions}

This is the first study to profile urogenital microbiota associated with the expression of PD-L1 in male patients with NMIBC. Our study shows that urogenital 


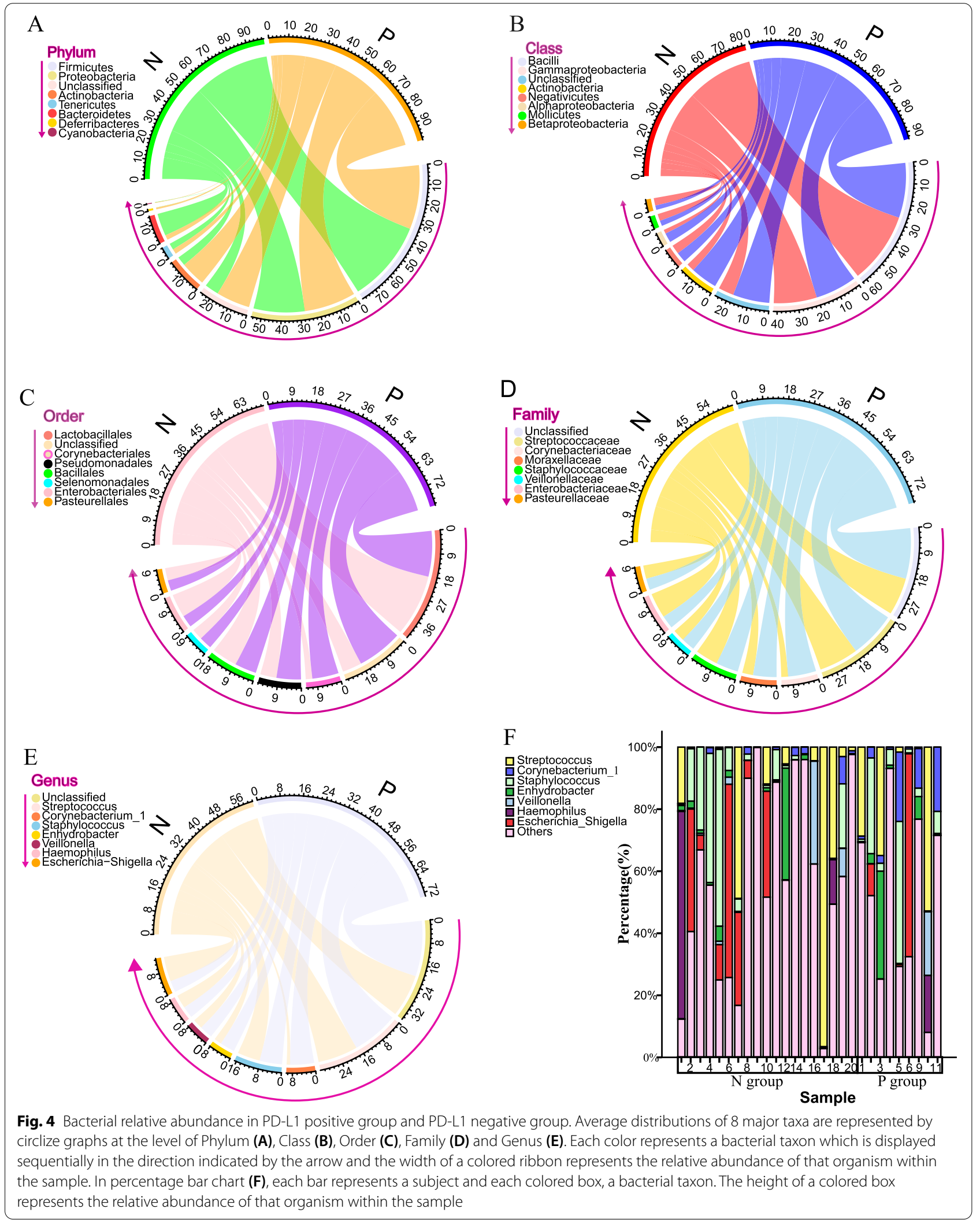




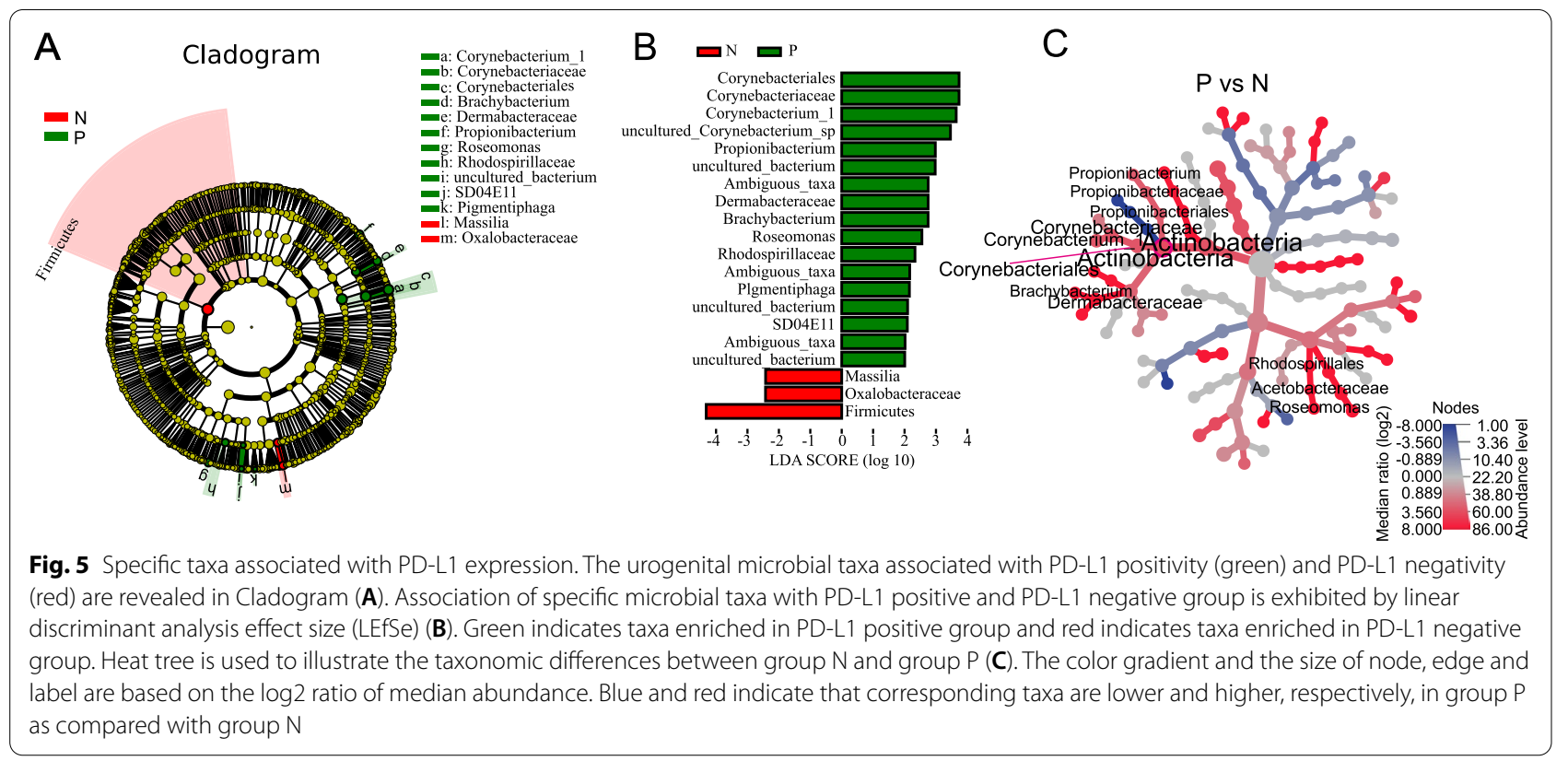

microbiota is associated with PD-L1 expression in male patients with NMIBC, but the exact relationship remains to be further investigated. Further exploration of the underlying mechanisms to explain these associations will offer new prospects for researches on tumor recurrence and application of PD1 or PD-L1 blockers in bladder cancer.

\section{Methods}

\section{Study design, subject recruitment and sample collection}

The aim of this cross-sectional study was to characterize urogenital microbiota associated with PD-L1 expression in male patients with NMIBC. Urine specimens and cancerous tissues were collected from male patients with NMIBC who were admitted to Nanfang Hospital in China between March 2017 and June 2019. Eventually, twenty-eight subjects histologically confirmed as non-muscle invasive bladder cancer, aged from 43 to 79 years, were enrolled for the study. The urine specimens were collected prior to the prescription of antibiotics and operation for bladder cancer. Exclusion criteria for the participants included prior known sexually transmitted infection, current urinary tract infection (based on urine dipstick or standard cultivation) or antibiotic consumption within 1 month for any reason. All participants were required to finish a questionnaire to collect their demographic and medical information. Following the Medicine Institutional Review Board of Southern Medical University approval (ethical code: NFEC-2020-045) and the Declaration of Helsinki principles, all participants gave written informed consent for their information, urine and tissue collection with analysis for research purposes.
All urine specimens for urinalysis were obtained by the mid-stream clean catch method with the guidance of urotherapy nurses, then immediately shipped on cold pack to laboratory within an hour for centrifugation at $16,000 \mathrm{~g}$ for $10 \mathrm{~min}$ at $4^{\circ} \mathrm{C}$. The precipitates were stored at -80 degrees Celsius until further processing.

\section{DNA Isolation and 16S rRNA Gene Sequencing}

To avoid contamination, DNA was extracted using the DNeasy Blood and Tissue Kit following the cultured cells protocol provided by the manufacturer (Qiagen, Germany) in a laminar flow hood. The Nanodrop ND-1000 spectrophotometer (Thermo Electron Corporation, USA) was used for the measurement of extracted DNA concentration. 16S rDNA sequences of the genomic DNA isolated from the urine specimens was amplified with PCR using primer sets specific for V3-V4 regions (314F: $5^{\prime}$-NCCT ACGGGNGGCWGCAG- $3^{\prime}$; and 805R: 5'NGACTAC HVGGGTATCTAATCC-3') [32]. We added extraction negative controls (no urine) and PCR negative controls (no template) to evaluate the presence of contaminating sequences in reagents. Unincorporated nucleotides and primers were eliminated using the Qiaquick PCR purification kit (Qiagen, Valencia, USA) to obtain the final PCR purified products [47]. After normalization to equal DNA concentration, the purified samples were sequenced using the Illumina Miseq sequencer (Illumina, USA). The $16 \mathrm{~S}$ rRNA gene sequence data reported in this paper have been deposited in the Genome Sequence Archive (Genomics, Proteomics \& Bioinformatics 2017) in BIG Data Center (Nucleic Acids Res 2018), Beijing Institute of Genomics 
(BIG), Chinese Academy of Sciences, under accession numbers CRA002985, CRA002985 that are publicly accessible at https://bigd.big.ac.cn/gsa.

\section{Immunohistochemistry}

Immunohistochemical detection of PD-L1 was performed on formalin-fixed paraffin-embedded tissues using a mouse monoclonal anti-PD-L1 antibody (405.9A11, dilution 1:200, Cell Signaling Technology) and following the protocol provided by the manufacturer [48]. For each sample, the membranous expression of PD-L1 in tumor cells or immune cells was determined and scored in blinded manner with respect to clinical data by two independent genitourinary pathologists. The tumor was considered positive for PD-L1 if $\geq 1 \%$ of tumor cells or immune cells had histologic evidence of plasma membrane staining. PD-L1 expression in tumor cells or immune cells was scored as the percentage of stained cells. And the intensity of PD-L1 was assessed and recorded as absent (0), mild $(1+)$, moderate $(2+)$ and severe $(3+)$.

\section{Statistical Analysis}

\section{Clinical Data Analysis}

Normal distributions of the data were estimated with Shapiro-Wilk test and homoscedasticity of variances was checked using Levene test. The differences between groups were tested using Student's t test for measurement data subject to normal distribution and homogeneity of variance and Fisher's Exact Test for counting data. While Mann-Whitney U test was used for the statistical analysis of ranked data or measurement data that did not conform to normal distribution or homogeneity test of variance. Statistical analysis was performed using SPSS statistics version 24 (IBM corp, Amonk, NY, USA). All tests were two sided and $P<0.05$ was considered statistically significant.

\section{Bioinformatics analysis}

In order to obtain clean reads, the raw data of $16 \mathrm{~S}$ rRNA gene sequences were filtered using QIIME [49] to eliminate reads with adapter pollution and low quality. Metadata are given in Supplementary Table S2. The paired-end reads were merged with their overlap using standardized bioinformatic pipeline to attain complete sequence which were clustered into operational taxonomic units (OTUs) with 97\% similarity and the representative sequences were picked by UPARSE [50-52]. Subsequently, UCHIME method in VSEARCH (v2.3.4) was used for the detection and removal of chimera $[53,54]$. The representative sequence from each clustered OTU was aligned to the SILVA database and the Greengenes database and classified with the RDP classifier to achieve species annotation [55-57].
Observed Species, Chao1, Ace, Shannon and Simpson indices were calculated using QIIME to evaluate alpha diversity. Among them, the Observed Species, Chao 1 and Ace indices are mainly used to assess the species richness of samples, while Shannon and Simpson indices mirror both species richness and evenness. The difference of alpha diversity between groups was evaluated by Mann-Whitney U Test (group number $=2$ ) or Kruskal-Wallis test (group number $>2$ ) using SPSS statistics version 24 (IBM corp, Amonk, NY, USA) or R software. The weighted UniFrac and unweighted UniFrac distances were calculated for the comparison of Beta-diversity of different groups. Three-dimensional plots were generated based on Principal coordinate analysis (PCoA) of these distance matrices in QIIME for the visual clustering of the bacterial communities. To statistically support the differences in beta-diversity between groups, these distances of different groups were compared using Adonis test or ANOSIM test.

Average distribution of major taxa was represented by circlize graphs which were drawn with the circlize package in $R$. To detect significantly different bacteria between groups, the relative abundance of bacteria were compared using Metastats. Taxa summaries were input into Linear discriminant analysis effect size (LEfSe) via the Huttenhower Lab Galaxy Server for further screening of significantly different bacteria between groups at different taxonomic level [58]. In the settings of LEfSe, the significantly specific bacteria were identified using the Mann-Whitney $U$ test, and their effect size were estimated via linear discriminant analysis (LDA) of which the threshold for discriminative features was 2.0. While heat tree was generated with the workflow provided by Chong et al. [59] and the use of MicrobiomeAnalyst (https://www.micro biomeanalyst.ca).

\section{Supplementary Information}

The online version contains supplementary material available at https://doi. org/10.1186/s12866-021-02407-8.

Additional file 1: Supplementary Table S1.The number of reads and OTUs for each sample.

Additional file 2: Supplementary Table S2. The information of Metadata.

Additional file 3: Figure S1. Alpha Diversity for group C (with mild PD-L1 expression) and group D (with moderate or severe PD-L1 expression). observed species (A), Chao1 index (B), Ace index (C), Shannon index (D) and Simpson index (E) were used for the assessment of alpha diversity between group $C$ and group D. We found that as the expression intensity of PD-L1 increased, the bacterial richness increased as well. Ns, not significant (based on $\mathrm{P}<0.05$ ).

Additional file 4: Figure S2. Alpha Diversity for group F (with $\geq 5 \%$ tumor cells membrane staining) and group $\mathrm{O}$ (with $<5 \%$ tumor cells membrane staining). observed species(A),Chao1 index (B),Ace index(C), Shannon 
index (D) and Simpson index (E) were used for the assessment of alpha diversity between group $\mathrm{F}$ and group $\mathrm{O}$. Urogenital microbiota of group $F$ had higher richness than that of group $\mathrm{O}$. Ns, not significant (based on $\mathrm{P}<0.05)$.

Additional file 5: Figure S3. Specific taxa associated with different PD-L1 expression levels. Association of specific microbial taxa with higher PD-L1 expression (group F, PD-L1 $1 \geq 5 \%$ ) and lower PD-L1 expression (group O, $1 \% \leq \mathrm{PD}-\mathrm{L} 1<5 \%$ ) is exhibited by linear discriminant analysis effect size (LEfSe).Green indicates taxa enriched in group $\mathrm{O}$ and red indicates taxa enriched in group $F$.

\section{Acknowledgments}

The authors are grateful to all the participants.

\section{Authors' contributions}

PW, CC, JZ: conception and design; CC, ZH, PH, KL, JRZ, YW, BL: acquisition of data; $\mathrm{CC}, \mathrm{PH}, \mathrm{KL}$ : analysis and interpretation of data; $\mathrm{CC}, \mathrm{ZH}$ : drafting of the manuscript; PW, JZ, JRZ: critical revision of the manuscript for important intellectual content; CC, PH, KL: statistical analysis; PW, JZ: obtaining funding; PW, JZ: supervision. All authors revised the draft and approved the final version of the manuscript.

\section{Funding}

This study was supported by the Natural Science Foundation of Guangdong Province (grant no. 2021A1515012262), the Natural Science Foundation of Guangdong Province (grant no. 2020A1515011339), the National Natural Science Foundation of China (grant no. 82173304) and the National Natural Science Foundation of China (grant no. 81870522).

\section{Availability of data and materials}

The 16S rRNA gene sequence data reported in this paper have been deposited in the Genome Sequence Archive (Genomics, Proteomics \& Bioinformatics 2017) in BIG Data Center (Nucleic Acids Res 2018), Beijing Institute of Genomics (BIG), Chinese Academy of Sciences, under accession numbers CRA002985, CRA002985 that are publicly accessible at https:// bigd.big.ac.cn/gsa.

\section{Declarations}

\section{Ethics approval and consent to participate}

This study was approved by the Medicine Institutional Review Board of Southern Medical University (NFEC-2020-045). All participants gave written informed consent for their information, urine and tissue collection with analysis for research purposes.

\section{Consent for publication}

Not applicable.

\section{Competing interests}

The authors declare that they have no competing interests.

\section{Author details}

${ }^{1}$ Department of Urology, Nanfang Hospital, Southern Medical University, Guangzhou, China. ${ }^{2}$ The third hospital of mianyang, Sichuan Province, China. ${ }^{3}$ Department of Urology, Meizhou People's Hospital, Meizhou, China. ${ }^{4}$ Meizhou hospital of TCM, Meizhou, China. ${ }^{5}$ School of Pharmaceutical Sciences, Southern Medical University, Guangzhou, China. ${ }^{6} \mathrm{Clinical}$ Microbiota Center, Nanfang Hospital, Southern Medical University, Guangzhou, China.

Received: 23 July 2021 Accepted: 29 November 2021

Published online: 04 January 2022

\section{References}

1. Bray F, Ferlay J, Soerjomataram I, Siegel RL, Torre LA, Jemal A. Global cancer statistics 2018: GLOBOCAN estimates of incidence and mortality worldwide for 36 cancers in 185 countries. CA Cancer J Clin. 2018;68(6):394-424.
2. de Braud F, Maffezzini M, Vitale V, Bruzzi P, Gatta G, Hendry WF, et al. Bladder cancer. Critical reviews in oncology/hematology. 2002;41(1):89-106.

3. Kamat AM, Hahn NM, Efstathiou JA, Lerner SP, Malmström PU, Choi W, et al. Bladder cancer. Lancet (London, England). 2016;388(10061):2796-810.

4. Fukumoto K, Kikuchi E, Mikami S, Hayakawa N, Matsumoto K, Niwa N, et al. Clinical Role of Programmed Cell Death-1 Expression in Patients with Non-muscle-invasive Bladder Cancer Recurring After Initial Bacillus Calmette-Guérin Therapy. Ann Surg Oncol. 2018;25(8):2484-91.

5. Hurwitz M, Adeniran A, Yao X, Hafez N, Schalper K, Rimm D, et al. The effect of BCG intravesical therapy and recurrence on PDL1 expression in non-invasive bladder cancers. J Clin Oncol. 2015;33:e15504.

6. Blank C, Gajewski TF, Mackensen A. Interaction of PD-L1 on tumor cells with PD-1 on tumor-specific T cells as a mechanism of immune evasion: implications for tumor immunotherapy. Cancer immunology, immunotherapy: Cll. 2005;54(4):307-14.

7. Shi L, Chen S, Yang L, Li Y. The role of PD-1 and PD-L1 in T-cell immune suppression in patients with hematological malignancies. Journal of hematology \& oncology. 2013;6(1):74.

8. Cha JH, Chan LC, Li CW, Hsu JL, Hung MC. Mechanisms Controlling PD-L1 Expression in Cancer. Molecular cell. 2019;76(3):359-70.

9. Sun C, Mezzadra R, Schumacher TN. Regulation and Function of the PD-L1 Checkpoint. Immunity. 2018;48(3):434-52.

10. Tang H, Liang Y, Anders RA, Taube JM, Qiu X, Mulgaonkar A, et al. PD-L1 on host cells is essential for PD-L1 blockade-mediated tumor regression. The Journal of clinical investigation. 2018;128(2):580-8.

11. Groeger S, Domann E, Gonzales JR, Chakraborty T, Meyle J. B7-H1 and B7-DC receptors of oral squamous carcinoma cells are upregulated by Porphyromonas gingivalis. Immunobiology. 2011;216(12):1302-10.

12. Groeger S, Jarzina F, Mamat U, Meyle J: Induction of B7-H1 receptor by bacterial cells fractions of Porphyromonas gingivalis on human oral epithelial cells: $\mathrm{B} 7-\mathrm{H} 1$ induction by Porphyromonas gingivalis fractions. Immunobiology 2017, 222(2):137-147.

13. Gollwitzer ES, Saglani S, Trompette A, Yadava K, Sherburn R, McCoy KD, et al. Lung microbiota promotes tolerance to allergens in neonates via PD-L1. Nature medicine. 2014;20(6):642-7.

14. Costello EK, Stagaman K, Dethlefsen L, Bohannan BJ, Relman DA. The application of ecological theory toward an understanding of the human microbiome. Science (New York, NY). 2012;336(6086):1255-62.

15. Brubaker L, Gourdine JF, Siddiqui NY, Holland A, Halverson T, Limeria R, Pride D, Ackerman L, Forster CS, Jacobs KM et al: Forming Consensus To Advance Urobiome Research. mSystems 2021, 6(4):e0137120.

16. Babjuk M, Burger M, Compérat EM, Gontero P, Mostafid AH, Palou J, et al. European Association of Urology Guidelines on Non-muscle-invasive Bladder Cancer (TaT1 and Carcinoma In Situ) - 2019 Update. European urology. 2019;76(5):639-57.

17. Zaghloul MS. Bladder cancer and schistosomiasis. Journal of the Egyptian National Cancer Institute. 2012;24(4):151-9.

18. Mostafa MH, Sheweita SA, O'Connor PJ. Relationship between schistosomiasis and bladder cancer. Clinical microbiology reviews. 1999;12(1):97-111.

19. Hicks RM, Ismail MM, Walters CL, Beecham PT, Rabie MF, El Alamy MA Association of bacteriuria and urinary nitrosamine formation with Schistosoma haematobium infection in the Qalyub area of Egypt. Transactions of the Royal Society of Tropical Medicine and Hygiene. 1982;76(4):519-27.

20. Pettenati $C$, Ingersoll MA. Mechanisms of BCG immunotherapy and its outlook for bladder cancer. Nature reviews Urology. 2018;15(10):615-25.

21. Markowski MC, Boorjian SA, Burton JP, Hahn NM, Ingersoll MA, Maleki Vareki S, et al. The Microbiome and Genitourinary Cancer: A Collaborative Review. European urology. 2019;75(4):637-46.

22. Wu P, Zhang G, Zhao J, Chen J, Chen Y, Huang W, et al. Profiling the Urinary Microbiota in Male Patients With Bladder Cancer in China. Frontiers in cellular and infection microbiology. 2018;8:167.

23. Zitvogel L, Daillère R, Roberti MP, Routy B, Kroemer G. Anticancer effects of the microbiome and its products. Nature reviews Microbiology. 2017;15(8):465-78.

24. Ma W, Zhang W, Shen L, Liu J, Yang F, Maskey N, et al. Can Smoking Cause Differences in Urine Microbiome in Male Patients With Bladder Cancer? A Retrospective Study. Frontiers in oncology. 2021;11:677605.

25. Dzutsev A, Goldszmid RS, Viaud S, Zitvogel L, Trinchieri G. The role of the microbiota in inflammation, carcinogenesis, and cancer therapy. European journal of immunology. 2015;45(1):17-31. 
26. Lynch SV, Pedersen O. The Human Intestinal Microbiome in Health and Disease. The New England journal of medicine. 2016;375(24):2369-79.

27. Willis AD, Minot SS. Strategies to Facilitate Translational Advances from Microbiome Surveys. Trends in microbiology. 2020;28(5):329-30.

28. Ribas A, Wolchok JD. Cancer immunotherapy using checkpoint blockade. Science (New York, NY). 2018;359(6382):1350-5.

29. Witjes JA, Bruins HM, Cathomas R, Compérat EM, Cowan NC, Gakis G, et al. European Association of Urology Guidelines on Muscle-invasive and Metastatic Bladder Cancer: Summary of the 2020 Guidelines. Eur Urol. 2021;79(1):82-104

30. Matson V, Fessler J, Bao R, Chongsuwat T, Zha Y, Alegre ML, et al. The commensal microbiome is associated with anti-PD-1 efficacy in metastatic melanoma patients. Science (New York, NY). 2018;359(6371):104-8.

31. Routy B, Le Chatelier E, Derosa L, Duong CPM, Alou MT, Daillère R, et al. Gut microbiome influences efficacy of PD-1-based immunotherapy against epithelial tumors. Science (New York, NY). 2018;359(6371):91-7.

32. Bi H, Tian Y, Song C, Li J, Liu T, Chen Z, et al. Urinary microbiota - a potential biomarker and therapeutic target for bladder cancer. Journal of medical microbiology. 2019;68(10):1471-8.

33. Eribe ER, Olsen I. Leptotrichia species in human infections. Anaerobe. 2008;14(3):131-7.

34. Castaño-Rodríguez N, Goh KL, Fock KM, Mitchell HM, Kaakoush NO. Dysbiosis of the microbiome in gastric carcinogenesis. Scientific reports. 2017;7(1):15957

35. Gao Z, Guo B, Gao R, Zhu Q, Qin H. Microbiota disbiosis is associated with colorectal cancer. Frontiers in microbiology. 2015;6:20.

36. Saffarian A, Mulet C, Regnault B, Amiot A, Tran-Van-Nhieu J, Ravel J, et al. Crypt- and Mucosa Associated Core Microbiotas in Humans and Their Alteration in Colon Cancer Patients. mBio. 2019:10(4):e01315-19.

37. Torres PJ, Fletcher EM, Gibbons SM, Bouvet M, Doran KS, Kelley ST. Characterization of the salivary microbiome in patients with pancreatic cancer. PeerJ. 2015;3:e1373.

38. Warren RL, Freeman DJ, Pleasance S, Watson P, Moore RA, Cochrane K, et al. Co-occurrence of anaerobic bacteria in colorectal carcinomas. Microbiome. 2013;1(1):16.

39. Jang JY, Song IS, Baek KJ, Choi Y, Ji S. Immunologic characteristics of human gingival fibroblasts in response to oral bacteria. Journal of periodontal research. 2017:52(3):447-57.

40. Trikha M, Corringham R, Klein B, Rossi JF. Targeted anti-interleukin-6 monoclonal antibody therapy for cancer: a review of the rationale and clinical evidence. Clinical cancer research : an official journal of the American Association for Cancer Research. 2003:9(13):4653-65.

41. Tanaka T, Narazaki M, Kishimoto T. IL-6 in inflammation, immunity, and disease. Cold Spring Harbor perspectives in biology. 2014;6(10):a016295.

42. Chan LC, Li CW, Xia W, Hsu JM, Lee HH, Cha JH, et al. IL-6/JAK1 pathway drives PD-L1 Y112 phosphorylation to promote cancer immune evasion. The Journal of clinical investigation. 2019:129(8):3324-38.

43. Calcinotto A, Brevi A, Chesi M, Ferrarese R, Garcia Perez L, Grioni M, et al. Microbiota-driven interleukin-17-producing cells and eosinophils synergize to accelerate multiple myeloma progression. Nature communications. 2018:9(1):4832.

44. Zhang M, Fan X, Fang B, Zhu C, Zhu J, Ren F. Effects of Lactobacillus salivarius Ren on cancer prevention and intestinal microbiota in 1 , 2-dimethylhydrazine-induced rat model. Journal of microbiology (Seoul, Korea). 2015:53(6):398-405.

45. Myles IA, Earland NJ, Anderson ED, Moore IN, Kieh MD, Williams KW, et al. First-in-human topical microbiome transplantation with Roseomonas mucosa for atopic dermatitis. JCI Insight. 2018;3(9):e120608.

46. Colliou N, Ge Y, Sahay B, Gong M, Zadeh M, Owen JL, et al. Commensal Propionibacterium strain UF1 mitigates intestinal inflammation via Th17 cell regulation. The Journal of clinical investigation. 2017;127(11):3970-86.

47. Wu P, Chen Y, Zhao J, Zhang G, Chen J, Wang J, et al. Urinary Microbiome and Psychological Factors in Women with Overactive Bladder. Frontiers in cellular and infection microbiology. 2017;7:488.

48. Inman BA, Sebo TJ, Frigola X, Dong H, Bergstralh EJ, Frank I, et al. PD-L1 (B7-H1) expression by urothelial carcinoma of the bladder and BCGinduced granulomata: associations with localized stage progression. Cancer. 2007;109(8):1499-505

49. Caporaso JG, Kuczynski J, Stombaugh J, Bittinger K, Bushman FD, Costello EK, et al. QIIME allows analysis of high-throughput community sequencing data. Nature methods. 2010;7(5):335-6.
50. Zhang J, Liu YX, Zhang N, Hu B, Jin T, Xu H, et al. NRT1.1B is associated with root microbiota composition and nitrogen use in field-grown rice. Nature biotechnology. 2019;37(6):676-84.

51. Magoč T, Salzberg SL. FLASH: fast length adjustment of short reads to improve genome assemblies. Bioinformatics (Oxford, England). 2011;27(21):2957-63

52. Edgar RC. UPARSE: highly accurate OTU sequences from microbial amplicon reads. Nature methods. 2013;10(10):996-8.

53. Rognes T, Flouri T, Nichols B, Quince C, Mahé F. VSEARCH: a versatile open source tool for metagenomics. PeerJ. 2016;4:e2584.

54. Edgar RC. Search and clustering orders of magnitude faster than BLAST. Bioinformatics (Oxford, England). 2010;26(19):2460-1.

55. Quast C, Pruesse E, Yilmaz P, Gerken J, Schweer T, Yarza P, et al. The SILVA ribosomal RNA gene database project: improved data processing and web-based tools. Nucleic acids research. 2013;41(Database:issue ):D590-596.

56. DeSantis TZ, Hugenholtz P, Larsen N, Rojas M, Brodie EL, Keller K, et al. Greengenes, a chimera-checked $16 \mathrm{~S}$ rRNA gene database and workbench compatible with ARB. Applied and environmental microbiology. 2006:72(7):5069-72.

57. Wang Q, Garrity GM, Tiedje JM, Cole JR. Naive Bayesian classifier for rapid assignment of rRNA sequences into the new bacterial taxonomy. Applied and environmental microbiology. 2007;73(16):5261-7.

58. Segata N, Izard J, Waldron L, Gevers D, Miropolsky L, Garrett WS, et al. Metagenomic biomarker discovery and explanation. Genome biology. 2011;12(6):R60.

59. Chong J, Liu P, Zhou G, Xia J. Using MicrobiomeAnalyst for comprehensive statistical, functional, and meta-analysis of microbiome data. Nature protocols. 2020;15(3):799-821.

\section{Publisher's Note}

Springer Nature remains neutral with regard to jurisdictional claims in published maps and institutional affiliations.

Ready to submit your research? Choose BMC and benefit from:

- fast, convenient online submission

- thorough peer review by experienced researchers in your field

- rapid publication on acceptance

- support for research data, including large and complex data types

- gold Open Access which fosters wider collaboration and increased citations

- maximum visibility for your research: over 100M website views per year

At BMC, research is always in progress.

Learn more biomedcentral.com/submissions 